\title{
Sinomenine activation of Nrf2 signaling prevents inflammation and cerebral injury in a mouse model of ischemic stroke
}

\author{
FANGFANG BI ${ }^{1}$, YIYONG ZHANG ${ }^{2}$, WENBO LIU $^{3}$ and KELIANG XIE ${ }^{2,4}$ \\ ${ }^{1}$ Department of Medicine, Xi'an Peihua University, Xi'an, Shaanxi 710125; ${ }^{2}$ Department of Neurosurgery, \\ Jinan Jiyang District People's Hospital, Jinan, Shandong 251401; ${ }^{3}$ Department of Intensive Care Medicine, \\ College of Anesthesiology, Weifang Medical University, Weifang, Shandong 261053; ${ }^{4}$ Department of Anesthesiology, \\ First Affiliated Hospital of Weifang Medical University (Weifang People's Hospital), Weifang, Shandong 261000, P.R. China
}

Received June 19, 2020; Accepted January 29, 2021

DOI: $10.3892 / \mathrm{etm} .2021 .10079$

\begin{abstract}
Sinomenine (SINO), which is used clinically to treat rheumatoid arthritis and neuralgia, is derived from the root and stems of Sinomenium acutum. SINO has been reported to exert analgesic, sedative and anti-inflammatory effects, and provides a protective role against shock and organ damage. Studies have suggested that SINO primarily exerts it anti-inflammatory function by inhibiting NF- $\mathrm{B}$ signaling. There is also evidence to indicate that SINO may regulate inflammation Nuclear factor (erythroid-derived 2)-like 2 (Nrf2) signaling. The present study aimed to investigate whether the anti-inflammatory and cerebral protective effects of SINO were induced through Nrf2 both in vitro and in vivo. The results revealed that SINO significantly upregulated Nrf2 protein expression levels, increased Nrf2 nuclear translocation and the upregulated the protein expression levels of downstream factors. The treatment of a middle cerebral artery occlusion model mice with SINO effectively reduced cerebral damage and inflammation, and restored the balance in cerebral oxidative stress. In addition, SINO treatment also promoted Nrf2-dependent
\end{abstract}

Correspondence to: Professor Wenbo Liu, Department of Intensive Care Medicine, College of Anesthesiology, Weifang Medical University, 7166 Baotong West Street, Weifang, Shandong 261053, P.R. China

E-mail: boboliucn@126.com

Professor Keliang Xie, Department of Anesthesiology, First Affiliated Hospital of Weifang Medical University (Weifang People's Hospital), 151 Guangwen Street, Weifang, Shandong 261000, P.R. China

E-mail: xiekeliang2009@hotmail.com

Abbreviations: SINO, sinomenine; MCAO, middle cerebral artery occlusion; OGD, oxygen and glucose deprivation; Nrf2, nuclear factor-erythroid 2-related factor; HO-1, heme oxygenase-1; NQO1, NAD(P)H: Quinoneoxidoreductase 1; Arg-1, arginase-1; NOS2, nitric oxide synthase 2; SOD, superoxide dismutase; GPx, glutathione peroxidase

Key words: sinomenine, nuclear factor-erythroid 2-related factor, microglia, cerebral protection, inflammation microglia M1/M2 polarization and inhibited the phosphorylation of $\mathrm{I} \kappa \mathrm{B} \alpha$ as well as $\mathrm{NF}-\kappa \mathrm{B}$ nuclear translocation. This revealed an important upstream event that contributed to its anti-inflammatory and cerebral tissue protective effects. In conclusion, the findings of the present study identified a novel pathway through which SINO may exert its anti-inflammatory and cerebral protective functions, and provided a molecular basis for the potential applications of SINO in the treatment of cerebral inflammatory disorders.

\section{Introduction}

Ischemic stroke is a common clinical cerebrovascular disease that is accompanied by significant disability and mortality, with survivors often suffering from various degrees of neurological dysfunction (1). The mechanism of cerebral ischemic injury involves a number of pathophysiological processes, with accumulating evidence suggesting that inflammation is involved $(2,3)$. Therefore, the regulation of inflammatory signaling may represent a potential treatment strategy for ischemic stroke.

Microglia are a type of glial cell, which are the resident macrophages of the brain (4). Microglia are the first and most important line of defense in the central nervous system (CNS). As the immune effector cells of the CNS, microglia have been demonstrated to serve an important role in the processes behind cerebral injury (5). For example, activated microglia have been found in the surrounding lesions of various neurodegenerative diseases, including Alzheimer's disease, Parkinson's disease, muscular amyotrophic lateral sclerosis and multiple sclerosis (6). Activated microglia can release a number of molecules, including cytotoxic substances such as nitric oxide (NO), oxygen free radicals and proteolytic enzymes, as well as inflammatory factors such as IL-1, TNF- $\alpha$ and IFN- $\gamma$, which are essential for the pathological process of cerebral ischemic injury (7). Microglia exist in a dynamic equilibrium between the pro-inflammatory (M1) and anti-inflammatory (M2) types, and the polarization state is associated with the local microenvironment (8). In vitro, M0 type microglia have been revealed to be induced to the M1 type through lipopolysaccharide or IFN- $\gamma$, or induced to the M2 type through IL-4, IL-10 and TGF- $\alpha$ (8). The excessive activation of M1 type microglia 
leads to the secretion of large amounts of inflammatory factors and free radicals, such as macrophage colony-stimulatory factor, TNF- $\alpha$, IL-1 and IL-6, which subsequently promote a major inflammatory response (5). Therefore, it remains necessary to avoid the occurrence of neuroinflammatory reactions by regulating the balance of the polarization state of M1 and M2 microglia.

Nuclear factor (erythroid-derived 2)-like 2 (Nrf2) is a major regulator of the cell defense response against chemical or oxidative stress (9). Nrf2 modulation is achieved by various proteins and signaling pathways at both the cytoplasmic and nuclear level (10). The pharmacological activation of Nrf2 has been suggested as a promising therapeutic approach for several chronic diseases, including Alzheimer's and Parkinson's diseases (11). Interestingly, a previous study revealed that sinomenine (SINO) provided protection against ischemic/reperfusion-associated liver damage in a heme oxygenase-1 (HO-1) dependent manner (12), indicating that SINO may exert its protective effects through the Nrf2 signaling pathway, since $\mathrm{HO}-1$ is mainly regulated by $\mathrm{Nrf} 2$ signaling (13).

SINO is an active alkaloid extracted from the Chinese medical plant, Sinomenium acutum (14). Previous studies have reported that SINO possesses anti-inflammatory and immunoregulatory properties, as well as exhibiting significant therapeutic efficacy for rheumatoid arthritis $(15,16)$. In addition, SINO is protective against various autoimmune and inflammation-associated diseases $(17,18)$. SINO has been found to modulate immune responses mainly through downregulating the expression of inflammation-related molecules, including NO, TNF- $\alpha$, IL-1 $\beta$ and prostaglandin E3, both in vitro and in vivo (19-21). Other studies have demonstrated that SINO exerts its anti-inflammatory role by inhibiting I $\kappa \mathrm{B}$ phosphorylation and subsequent $\mathrm{NF}-\kappa \mathrm{B}$ transcription $(22,23)$. However, the underlying mechanism of action behind the protective effect of SINO in cerebral ischemic injury remains unclear. Therefore, the present study aimed to investigate the role of SINO and its potential mechanism of action in cerebral ischemic injury. The results indicated that SINO may inhibit neuroinflammation by targeting the microglia Nrf2 signaling pathway, both in vivo and in vitro, which provided a novel target for the treatment of ischemic stroke.

\section{Materials and methods}

Chemicals and regents. The following antibodies were used in the present study: SINO (MedChemExpress); ML385 (MedChemExpress); Nrf2, Keap1, NAD(P)H: Quinoneoxidoreductase (NQO1), Lamin B and HO-1 antibodies (Abcam); $\beta$-actin antibodies (Bioworld Technology, Inc.); NF- $\kappa \mathrm{B}$ p 65, Phosphorylated (p-)I $\mathrm{B} \alpha$ and $\mathrm{I} \kappa \mathrm{B} \alpha$ antibodies (Cell Signaling Technology, Inc.); HRP-conjugated secondary antibodies (Bioworld Technology, Inc.); and Dylight488 conjugated goat anti-rabbit IgG secondary antibodies (Bioworld Technology, Inc.).

Cell culture and treatment. BV2 cells (Shanghai Kang Lang Biological Technology Co. Ltd.) were cultured in DMEM (Gibco; Thermo Fisher Scientific, Inc.) supplemented with 10\% FBS (Gibco; Thermo Fisher Scientific, Inc.) and maintained at $37^{\circ} \mathrm{C}$ in a $5 \% \mathrm{CO}_{2}$ incubator (Thermo Fisher Scientific, Inc.). Oxygen glucose deprivation (OGD) was induced according to a previously described method (24). BV2 cells were cultured at $37^{\circ} \mathrm{C}$ with deoxygenated DMEM without glucose and FBS, in the presence of a premixed gas $\left(1 \% \mathrm{O}_{2}, 94 \% \mathrm{~N}_{2}\right.$ and $5 \% \mathrm{CO}_{2}$ ) for $4 \mathrm{~h}$. The cells were subsequently cultured in normal DMEM supplemented with 10\% FBS and maintained at $37^{\circ} \mathrm{C}$ in a $5 \% \mathrm{CO}_{2}$ incubator. Cells in the control group were cultured with normal DMEM and 10\% FBS throughout.

Cell viability analysis. BV2 cells were cultured on a 96-well plate and treated with increasing concentrations $(0-200 \mu \mathrm{M})$ of SINO for $24 \mathrm{~h}$. Cell viability was then measured using a Cell Counting Kit-8 (ProteinTech Group, Inc.) according to the manufacturer's instructions. In total, $1 \mathrm{~h}$ was the duration of the incubation with the CCK- 8 reagent at $37^{\circ} \mathrm{C}$.

Establishment of middle cerebral artery occlusion (MCAO). The present study was approved by the Ethics Committee of the Medical College of Xi'an Peihua University. A total of 48 male C57BL/6 mice (age, 12 weeks; weight, $25 \pm 2 \mathrm{~g}$ ) were obtained from Xi'an Jiaotong University Experimental Animal Center. They were housed in the specific pathogen-free conditions with standard temperature $\left(22 \pm 1^{\circ} \mathrm{C}\right)$, humidity $(50-60 \%)$ and light conditions ( $12 \mathrm{~h}$ light/dark cycle), with access to food and water ad libitum.

MCAO surgery was performed as previously described (25). Mice were anesthetized with an intraperitoneal injection of $5 \%$ chloral hydrate $(400 \mathrm{mg} / \mathrm{kg})$. A silicone-coated 6-0 suture (Covidien; Medtronic, Ltd.) was slowly inserted from the exposed external carotid artery to the internal carotid artery and wedged into the circle of Willis to obstruct the opening of the middle cerebral artery. The distance from the bifurcation of the internal/external carotid artery to the middle cerebral artery was $9 \pm 1.0 \mathrm{~mm}$. The suture was withdrawn following $60 \mathrm{~min}$ of obstruction. Mice were divided into four groups $(n=6)$ as follows: i) The sham group that was identical with MCAO but did not include the occlusion of the middle cerebral artery; ii) SINO group that received SINO at a dose of $20 \mathrm{mg} / \mathrm{kg}$ daily for 3 days; iii) MCAO group; and iv) SINO/MCAO group in which SINO was subsequently injected intraperitoneally into mice $6 \mathrm{~h}$ after MCAO surgery at a dose of $20 \mathrm{mg} / \mathrm{kg}$ daily for 3 days. Notably, $20 \mathrm{mg} / \mathrm{kg}$ SINO used were performed as previously described $(26,27)$. The sham group received the same volume of saline intraperitoneally. After completion of the experiment, mice were sacrificed by exsanguination under deep anesthesia (sodium pentobarbital intraperitoneal injection, $50 \mathrm{mg} / \mathrm{kg}$ ). Death was confirmed by cessation of the heartbeat.

Histological analysis. For histological analysis, brain tissues were fixed in $4 \%$ paraformaldehyde overnight at $4^{\circ} \mathrm{C}$ and embedded in paraffin. Paraffin-embedded tissues were cut into $4-\mu \mathrm{m}$-thick sections and stained with H\&E using standard procedures (28). Stained sections from 6 animals were visualized in 10 randomly selected fields of view using a light microscope with x20 magnification (Nikon Corporation).

Brain water content measurement. Mice were deeply anesthetized and decapitated 3 days following MCAO surgery. Brain 
edema was analyzed according to a previously described method (29). Wet brains were weighed and then immediately dried at $95^{\circ} \mathrm{C}$ overnight. The brain water content was calculated using the following formula: [(Wet tissue weight-dry tissue weight)/wet tissue weight] x100\%.

Measurement of cerebral antioxidant enzyme activities. The activities of cerebral enzymes, glutathione (GSH) peroxidase (GPx) and superoxide dismutase (SOD), were measured using their respective colorimetric assay kits (Abcam), according to the manufacturers' protocols. Briefly, for the GPx assay, the tissue homogenates were incubated with GSH reductase (GR), $\mathrm{GSH}$, tert-butyl hydroperoxide and NADPH at $25^{\circ} \mathrm{C}$ for $3 \mathrm{~min}$ and the absorbance was measured at wavelength of $340 \mathrm{~nm}$. In this assay, the generated GSH disulfide was reduced to GSH following the consumption of NADPH by GR. GPX activity was proportional to the decrease of NADPH (which is measured at $340 \mathrm{~nm}$ ). For the SOD assay, tissue homogenates were treated with water-soluble tetrazolium at $37^{\circ} \mathrm{C}$ for $30 \mathrm{~min}$ and the absorbance was measured at a wavelength of $560 \mathrm{~nm}$. In this assay, the water-soluble tetrazolium was converted by the superoxide radical anion to a formazan dye. SOD in the tissue lysates reduced $\mathrm{O}_{2}$-levels, thereby decreasing the dye formation.

Reverse transcription-semi-quantitative PCR (RT-sqPCR). Total RNA was extracted from the tissues or BV2 cells using TRIzol ${ }^{\circledR}$ reagent (Invitrogen; Thermo Fisher Scientific, Inc.) according to the manufacturer's protocol. Total RNA was reverse transcribed into cDNA using a Reverse Transcriptase-kit (Qiagen, Inc.), according to the manufacturer's protocol. The RT-sqPCR was performed using a 2x Taq Plus MasterMix (Cwbiotech, Inc.) and products were analyzed on $1 \%$ agarose gel and the intensity of each band was quantified by Image J software version 1.8.0.112 (National Institutes of Health). qPCR was subsequently performed using a SuperReal PreMix Plus (SYBR-Green) kit (Qiagen, Inc.) on a CFX96 ${ }^{\mathrm{TM}}$ Real-Time PCR Detection System (Bio-Rad Laboratories, Inc.). The thermocycling conditions for the sqPCR reaction included an initial step of $15 \mathrm{~min}$ at $95^{\circ} \mathrm{C}$ to activate the chemically modified hot-start Taq DNA polymerase, followed by 40 cycles of a $15-\mathrm{sec}$ denaturation at $95^{\circ} \mathrm{C}$ and then $30 \mathrm{sec}$ annealing and extension at $60^{\circ} \mathrm{C}$. GAPDH was used as the internal reference control. Primer sequences were as follows $(28,30)$ : TNF $\alpha$ forward, 5'-CATCTTCTCAAAATTCGAGT GAC-3' and reverse, 5'-TGGGAGTAGACAAGGTACAACCC-3'; IL-1 forward, 5'-TGGAAAAGCGGTTTGTCTTC-3' and reverse, 5'-TACCAGTTGGGGAACTCTGC-3'; NO synthase 2 (NOS2) forward, 5'-CAGCTGGGCTGTACAAACCT T-3' and reverse, 5'-CATTGGAAGTGAAGCGTTTCG-3'; IL-6 forward, 5'-GCTGG-TGACAACCACGGCCT-3' and reverse, 5'-AGCCTCGACTTGTGAAGTGGT-3'; IL-10 forward, 5'-GCTCTTACTGACTGGCATGAG-3' and reverse, 5'-CGC AGCTCTAGGAGCATGTG-3'; arginase-1 (Arg-1) forwards, 5'-GTGAAGAACCCACGGTCTGT-3' and reverse, 5'-GCC AGAGATGCTTCCAACTG-3'; GAPDH forwards, 5'-GGC CCGGTGCTGAGTATGTC-3' and reverse, 5'-TGCCTG CTTCACCACCTTCT-3'. Relative expression levels were analyzed using the $2^{-\Delta \Delta \mathrm{Cq}}$ method (31).
Western blotting. Protein was extracted with RIPA Lysis Buffer (Beyotime Institute of Biotechnology) from the tissues and cells, and quantified using a BCA protein assay kit (Thermo Scientific, Inc.). The cytoplasmic/nuclear fractions were separated using NE-PER Nuclear and Cytoplasmic Extraction Reagents (Thermo Fisher Scientific, Inc.) according to the manufacturer's manual. Proteins $(40 \mu \mathrm{g})$ were separated via $12 \%$ SDS-PAGE and transferred to PVDF membranes, which were blocked with $5 \%$ bovine serum albumin (Sigma-Aldrich; Merck $\mathrm{KGaA}$ ) for $2 \mathrm{~h}$ at room temperature. The membranes were then incubated at $4^{\circ} \mathrm{C}$ overnight with the following primary antibodies: Anti- $\beta$-actin (cat. no. AP0060, 1:5,000), anti-Nrf2 (cat. no. ab92946, 1:1,000), anti-NQO1 (cat. no. ab80588, 1:1,000), anti-Lamin B (cat. no. ab194109, 1:1,000), anti-HO-1 (cat. no. ab68477, 1:2,000), anti-NF-кB p65 (cat. no. 8242

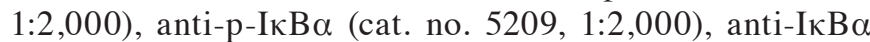
(cat. no. 4812 1:1,000). Following the primary antibody incubation, the membranes were washed with TBST $(0.1 \%$ Tween 20) and incubated with a HRP-conjugated secondary antibody (cat. no. BS13278, 1:5,000) for $1 \mathrm{~h}$ at room temperature. Protein bands were visualized using an ECL Plus Western Blotting Detection system (Bio-Rad Laboratories, Inc.).

Immunofluorescence staining. BV2 cells were cultured at $37^{\circ} \mathrm{C}$ in a 24-well chamber and treated with SINO and/or OGD for $4 \mathrm{~h}$. Immunofluorescence staining of $\mathrm{NF}-\kappa \mathrm{B}$ (cat. no. 8242, 1:500; Abcam) and subsequent nuclear staining with DAPI (2 $\mu \mathrm{g} / \mathrm{ml}$; Sigma-Aldrich; Merck KGaA) were performed as previously described (32). Briefly, the cells were fixed with $4 \%$ paraformaldehyde for $30 \mathrm{~min}$ and then permeabilized in PBS containing 2\% Triton X-100 for $5 \mathrm{~min}$ at room temperature. The cells were blocked with $5 \%$ bovine serum albumin (Sigma-Aldrich; Merck KGaA) for $2 \mathrm{~h}$ at room temperature and subsequently incubated with the anti-NF- $\kappa \mathrm{B}$ antibody at $4^{\circ} \mathrm{C}$ overnight and a DyLight 594-conjugated AffiniPure donkey anti-rabbit IgG secondary antibody (cat. no. BS10030, 1:100) at $1 \mathrm{~h}$ room temperature. The cells were also stained with DAPI for 5 min and observed under a confocal microscope with $\mathrm{x} 40$ magnification.

Statistical analysis. All data are presented as the mean \pm SEM and statistical analysis was performed using SPSS Version 18.0 (SPSS, Inc.). Each experiment was repeated in triplicate and statistical differences between 2 groups were analyzed using a two-tailed Student's t-test, while statistical differences between $>2$ groups were determined using one-way ANOVA followed by Tukey's post-hoc tests. $\mathrm{P}<0.05$ was considered to indicate a statistically significant difference.

\section{Results}

SINO treatment relieves MCAO-induced cerebral injuries. The cerebral protective role of SINO in a MCAO model mice was analyzed. Briefly, $20 \mathrm{mg} / \mathrm{kg}$ SINO was injected intraperitoneally into mice for 3 days following MCAO surgery, after which the cerebral histological changes and the brain water content were determined. $\mathrm{H} \& \mathrm{E}$ staining revealed that mice in the sham and SINO treatment groups did not have altered cerebral morphology or brain water content, while the brains of MCAO model mice exhibited a marked reduction 


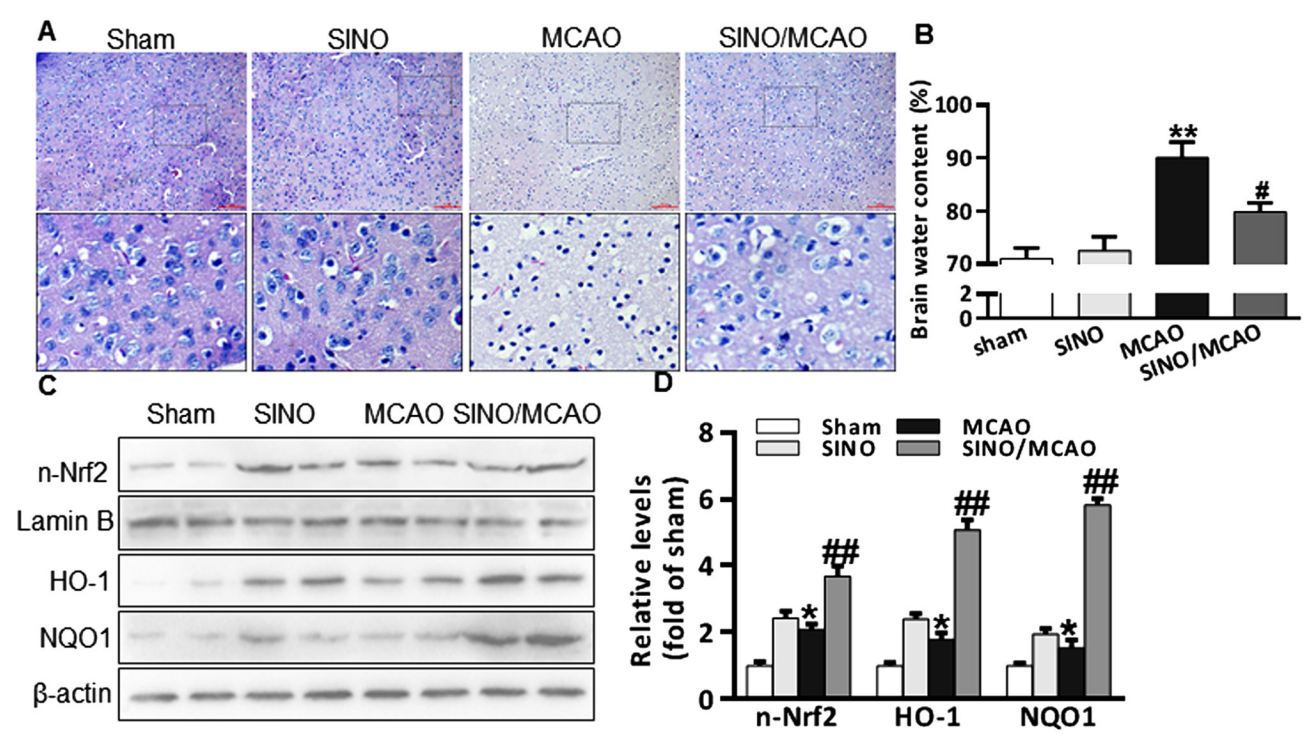

Figure 1. SINO treatment relieves MCAO-induced cerebral injuries. (A) Representative micrographs of H\&E staining of brain sections (x20 magnification; scale bar, $50 \mu \mathrm{m}$ ). (B) Brain water content analysis in brain tissues from Sham (sham-operated, $\mathrm{n}=6$ ), SINO (treatment only, $\mathrm{n}=6$ ), MCAO (MCAO-operated, $\mathrm{n}=6$ ) and SINO/MCAO (MCAO operated plus SINO treatment, $n=6$ ). (C) Western blot analysis of n-Nrf2, HO-1 and NQO1 protein expression levels from brain tissue. The samples from two randomly selected brains in each group were presented. (D) Quantification of brain protein expression levels. All the experiments were repeated at least three times. The data are presented as the mean \pm SEM. ${ }^{*} \mathrm{P}<0.05$ and ${ }^{* *} \mathrm{P}<0.01$ vs. Sham; ${ }^{\#} \mathrm{P}<0.05$ and ${ }^{\# \#} \mathrm{P}<0.01$ vs. MCAO. HO-1, heme oxygenase-1; MCAO, middle cerebral artery occlusion; n-Nrf2, nuclear-nuclear factor-erythroid 2-related factor; NQO1, NAD(P)H: Quinoneoxidoreductase 1; SINO, sinomenine.

in the number of nerve cells, as well as an increased brain water content (Fig. 1A and B). Notably, treatment with SINO following MCAO reduced the degree of pathological damage in the cerebral tissue, as well as the brain water content (Fig. 1A and B). A previous study reported that SINO-induced HO-1 serves an important role in attenuating cold ischemia/reperfusion injury in rats (12) and that HO-1 is an Nrf2 target gene (33). As such, the translocation of nuclear of Nrf2 and the expression levels of HO-1 and NQO1 were investigated. Compared with the sham group, Nrf2 translocation and HO-1 and NQO1 expression levels were increased following MCAO surgery. This result was and further enhanced by SINO treatment (Fig. 1C and D). These results indicated that SINO may protect against cerebral injury in MCAO model mice and the protective effect may be related to the activation of the Nrf2 signaling pathway.

SINO treatment activates the Nrf2 signaling pathway. To investigate the effect of SINO treatment on Nrf2 signaling, microglia BV2 cells were treated with 50-200 $\mu \mathrm{M}$ SINO for various durations (2-24 h) and the levels of HO-1 and NQO1 were analyzed using western blotting. As presented in Fig. 2A and B, SINO treatment significantly upregulated HO-1 and NQO1 expression levels in a concentration- and time-dependent manner. Nrf2 nuclear translocation is a necessary step for Nrf2 activation (30). SINO treatment markedly induced Nrf2 nuclear accumulation after $4 \mathrm{~h}$ (Fig. 2C). SINO treatment also upregulated the mRNA expression of HO-1 and NQO1 in a dose-dependent manner (Fig. 2D), suggesting that SINO may upregulate HO-1 and NQO1 at the transcriptional or post-transcription level. The cytotoxic effects of SINO were analyzed using a Cell Counting Kit 8 assay, which revealed that treatment with $\leq 200 \mu \mathrm{M}$ SINO for $24 \mathrm{~h}$ was unable to induce cell death, with no obvious changes in the amount of cell death following treatment with between 0-200 $\mu \mathrm{M}$ SINO
(Fig. 2E). Taken together, the results suggested that SINO may be an activator of the Nrf2 signaling pathway in microglial BV2 cells.

SINO treatment mitigates MCAO-associated inflammation and oxidative stress. Cerebral inflammation and oxidative stress are critical pathological processes associated with cerebral pathogenesis in MCAO model mice (34). MCAO model mice had a markedly upregulated expression of TNF- $\alpha$ and IL-1 $\beta$ compared with the sham group. However, SINO treatment significantly inhibited the production of both TNF- $\alpha$ and IL-1 $\beta$ (Fig. 3A). NOS2 is not only considered to be a pro-inflammatory mediator, but also the key enzyme that increases peroxynitrite production, a major reactive oxygen species (35). The brains of MCAO model mice had significantly upregulated mRNA expression levels of NOS2 (Fig. 3B). SOD and GPx are two important antioxidant enzymes (36). The enzymatic activities of SOD and GPx in the brains of MCAO model mice were found to be decreased (Fig. 3C and D); however, treatment with SINO significantly reversed the upregulation of NOS2 mRNA expression levels (Fig. 3B) and the reduction in SOD and GPx enzyme activities (Fig. 3C and D). Collectively, these results indicated that SINO may exert strong anti-inflammatory and anti-oxidative stress functions in MCAO model mice.

SINO treatment regulates microglia polarization and inflammation in an Nrf2-dependent manner. Although SINO exerts strong anti-inflammatory properties, its regulatory role in microglial polarization has not been fully determined. Thus, whether SINO affected the expression levels of M1 markers (NOS2 and IL-6) and M2 markers (Arg-1 and IL-10) in BV2 cells was investigated. The results demonstrated that OGD upregulated the expression levels of M1 markers, while SINO treatment significantly inhibited the OGD-induced increases 
A

B

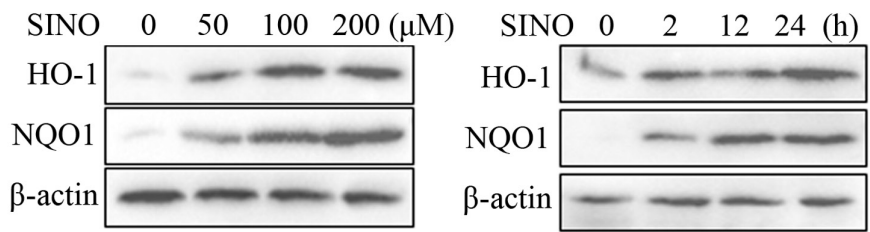

C SINO $00200(\mu \mathrm{M})$

$\square$ HO-1
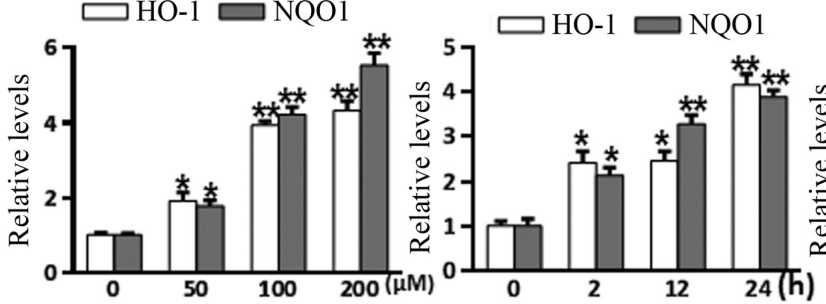

$n-\mathrm{Nrf} 2$

Lamin B

$\mathrm{t}-\mathrm{Nrf} 2$

$\beta$-actin

$0 \mathrm{n}-\mathrm{Nrf2}$

D

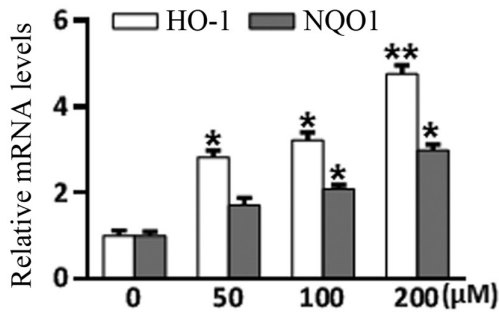

E

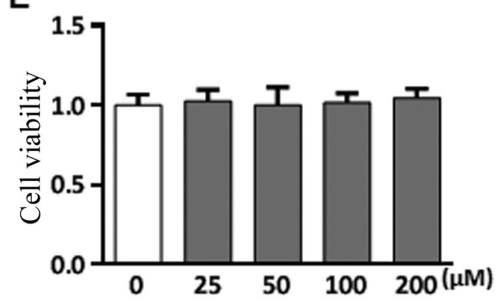

Figure 2. SINO treatment activates the Nrf2 signaling pathway. (A) BV2 cells were treated with a serial of doses of SINO for $24 \mathrm{~h}$. HO-1 and NQO1 protein expression levels were assayed using western blotting. (B) BV2 cells were treated with SINO $(200 \mu \mathrm{M})$ for various durations. HO-1 and NQO1 protein expression levels were assayed using western blotting. (C) BV2 cells were treated with SINO $(200 \mu \mathrm{M})$ for $4 \mathrm{~h}$. n-Nrf2 and t-Nrf2 protein expression levels were assayed using western blotting. Nuclear protein Lamin B and $\beta$-actin were used as controls. (D) BV2 cells were treated with serial doses of SINO for $24 \mathrm{~h}$. The mRNA expression levels of HO-1 and NQO1 were measured using reverse transcription-semi-quantitative PCR. (E) Cell viability assay. BV2 cells were treated with various doses of SINO for $24 \mathrm{~h}$. The cell viability was assayed by a CCK- 8 kit. All the experiments were repeated at least three times. " $\mathrm{P}<0.05$ and ${ }^{* * *} \mathrm{P}<0.01 \mathrm{vs}$. Control HO-1, heme oxygenase-1; NQO1, NAD(P)H: Quinoneoxidoreductase 1; n-, nuclear; Nrf2; nuclear factor-erythroid 2-related factor; SINO, sinomenine; t-, total.

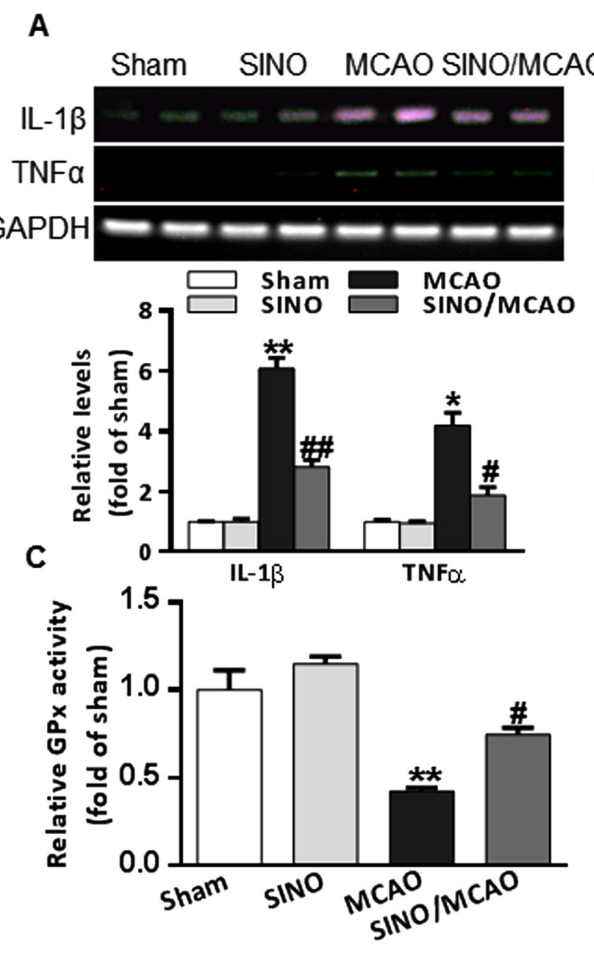

\section{B}
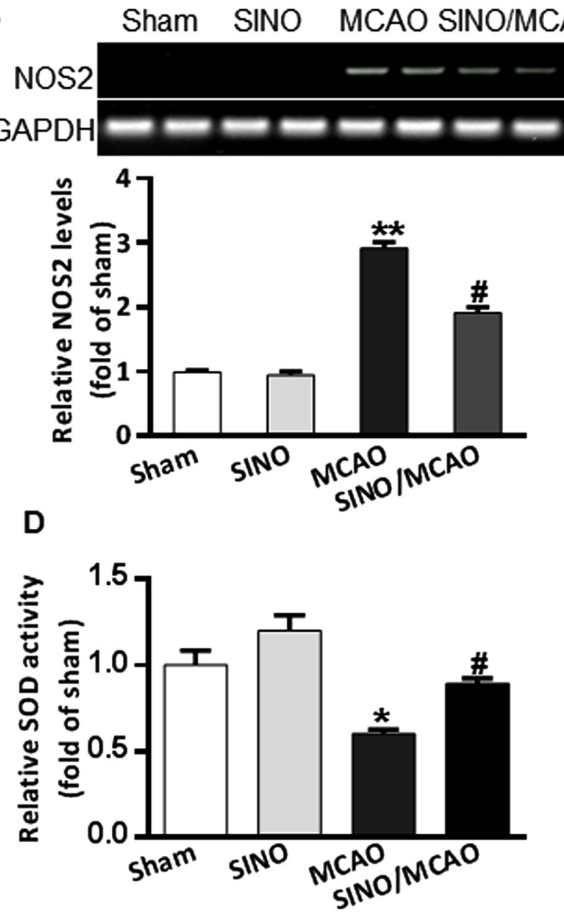

Figure 3. SINO treatment mitigates MCAO-associated inflammation and oxidative stress. (A) RT-sqPCR analysis of TNF- $\alpha$ and IL-1 $\beta$ mRNA expression levels in brain tissues. The results of two samples from each group were presented. (B) RT-sqPCR analysis of NOS2 mRNA expression levels in brain tissues. (C) The relative enzyme activities of GPx from the brain tissue of the four groups. (D) The relative enzyme activities of SOD from the brain tissue of four groups. All experiments were repeated at least three times. The data are presented as the mean $\pm \mathrm{SEM}$. " $\mathrm{P}<0.05$ and ${ }^{* *} \mathrm{P}<0.01 \mathrm{vs}$. Sham; ${ }^{*} \mathrm{P}<0.05$ and ${ }^{\# \#} \mathrm{P}<0.01$ vs. MCAO. GPx, glutathione peroxidase; MCAO, middle cerebral artery occlusion; NOS2, nitric oxide synthase 2; RT-sqPCR, reverse transcription-semi-quantitative PCR; SINO, sinomenine; SOD, superoxide dismutase. 

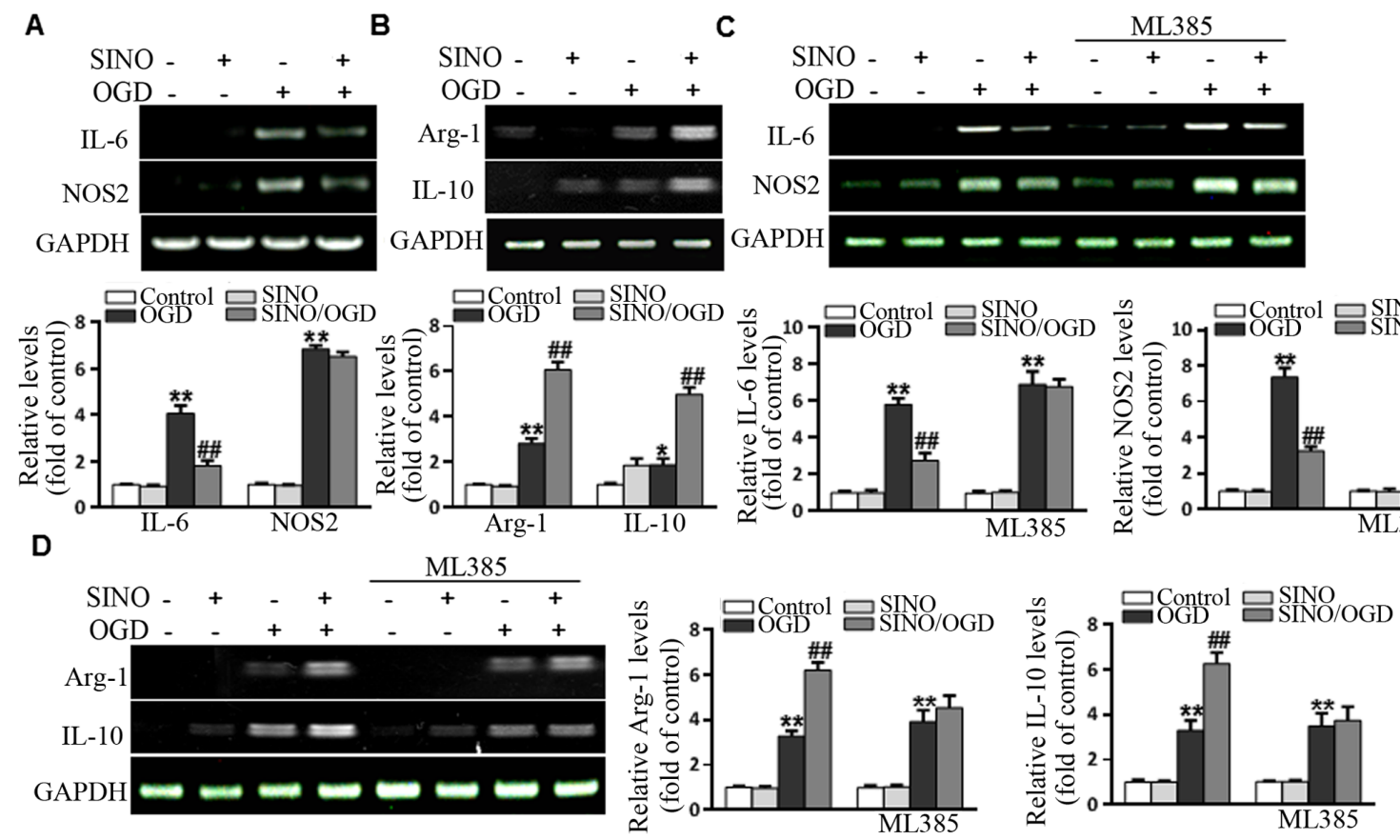

Figure 4. SINO treatment regulates microglia polarization and inflammation in an Nrf2-dependent manner. BV2 cells were stimulated with OGD for $4 \mathrm{~h}$ followed by treatment with SINO $(200 \mu \mathrm{M})$ for $12 \mathrm{~h}$. The mRNA expression levels of (A) M1 markers (IL-6/NOS2) and (B) M2 markers (IL-10/Arg-1) were determined using RT-sqPCR. BV2 cells were pretreated with ML385 $(5 \mu \mathrm{M})$ for $48 \mathrm{~h}$ to inhibit Nrf2 expression. Cells were then stimulated with OGD for $4 \mathrm{~h}$ followed by treatment with SINO $(200 \mu \mathrm{M})$ for $12 \mathrm{~h}$. The mRNA expression levels of (C) M1 and (D) M2 markers were measured using RT-sqPCR. The data are presented as the mean $\pm \mathrm{SEM}$ of three independent experiments. ${ }^{*} \mathrm{P}<0.05$ and ${ }^{* *} \mathrm{P}<0.01$ vs. Control; ${ }^{\# \#} \mathrm{P}<0.01$ vs. OGD. Arg-1, arginase-1; NOS2, nitric oxide synthase 2; OGD, oxygen and glucose deprivation; SINO, sinomenine; RT-sqPCR, reverse transcription-semi-quantitative PCR.

in IL-6 and NOS2 levels (Fig. 4A). In addition, OGD upregulated the expression levels of M2 markers, while SINO treatment markedly enhanced the OGD-induced expression of Arg-1 and IL-10 compared with the control group (Fig. 4B), suggesting that SINO may have dual functions in regulating microglial polarization. To further determine whether SINO exerted its effects through Nrf2, BV2 cells were treated with $5 \mu \mathrm{M}$ of the Nrf2 inhibitor, ML385, for 48 h. As shown in Fig. 4C and D, the effect of SINO treatment on the OGD-induced upregulation of NOS2, IL-6, Arg-1 and IL-10 expression levels was markedly reduced following the inhibition of Nrf2. In addition, the SINO-induced inhibition of OGD-induced I $\kappa \mathrm{B} \alpha$ phosphorylation (Fig. 5A) and NF- $\kappa$ B p65 nuclear translocation (Fig. 5B) was also markedly reduced following the inhibition of Nrf2, suggesting that Nrf2 signaling may be a crucial upstream event involved in mediating the SINO-induced inhibition of NF- $\mathrm{KB}$ signaling and inflammatory responses.

\section{Discussion}

The present study used MCAO model mice to establish an in vivo model of ischemic stroke to investigate the role of Nrf2 signaling in SINO-induced cerebral protection. The results of the present study revealed that SINO served dual functions in regulating inflammatory responses in microglia, in that SINO downregulated NOS2 and IL-6 expression levels and promoted Arg-1 and IL-10 expression levels. This subsequently suppressed NF- $\kappa B$ signaling, suggesting that the SINO-induced activation of Nrf2 may act upstream of its inhibition of NF- $\mathrm{KB}$ signaling.
Stroke is the main cause of disability in adults worldwide (37). Ischemic stroke accounts for $85 \%$ of strokes $(38,39)$ and is a clinical syndrome which leads to neurological deficits due to the ischemic and anoxic necrosis of the local tissue (40). Inflammation is an important response in the pathological process of ischemic stroke. Previous studies have reported that inflammation is the main factor that determines the outcome and long-term prognosis of patients with ischemic strokes (41). Microglia are macrophages that are widespread throughout the CNS and participate in the cellular immune process (42). At present, therapies that inhibit the M1 polarization of microglia cells through physical and pharmaceutical intervention, to promote anti-inflammatory functions, have been applied in the clinic (43). Physical methods to inhibit M1 polarization include electro-acupuncture guidance and hyperbaric oxygen treatment. Pharmaceutical interventions include exenatide acetate and minocycline. Exenatide acetate has been found to promote the change of microglia from the M1 to M2 type $(44,45)$. Minocycline has been found to inhibit the polarization of microglia to the M1 type, but has no effect on the M2 type (46-48). Kata et al (49) reported that rosuvastatin inhibits the proliferation and adhesion of microglia and upregulates the expression levels of certain anti-inflammatory genes, including $\mathrm{C}-\mathrm{X}-\mathrm{C}$ motif chemokine ligand $1, \mathrm{C}-\mathrm{C}$ motif chemokine ligand 5 and mannose binding lectin 2 . Concurrently, rosuvastatin also downregulated the expression levels of the pro-inflammatory cytokines, IL-1 $1 \beta$ and TNF- $\alpha$, upregulates the expression levels of the anti-inflammatory factor, IL-10, and promotes the polarization of microglia to the M2 type. The results of the present study confirmed that SINO treatment effectively inhibited the OGD-induced 
A
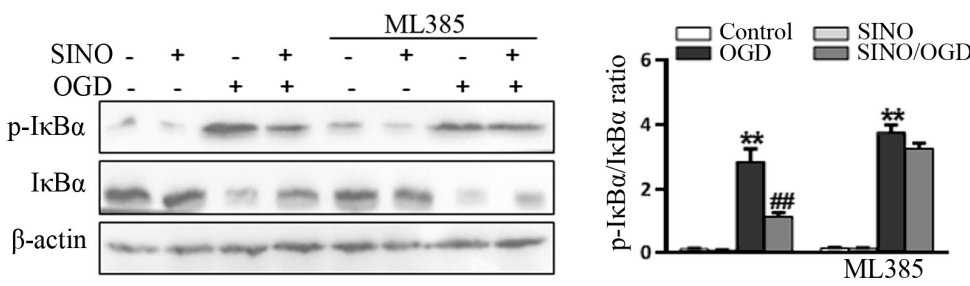

B

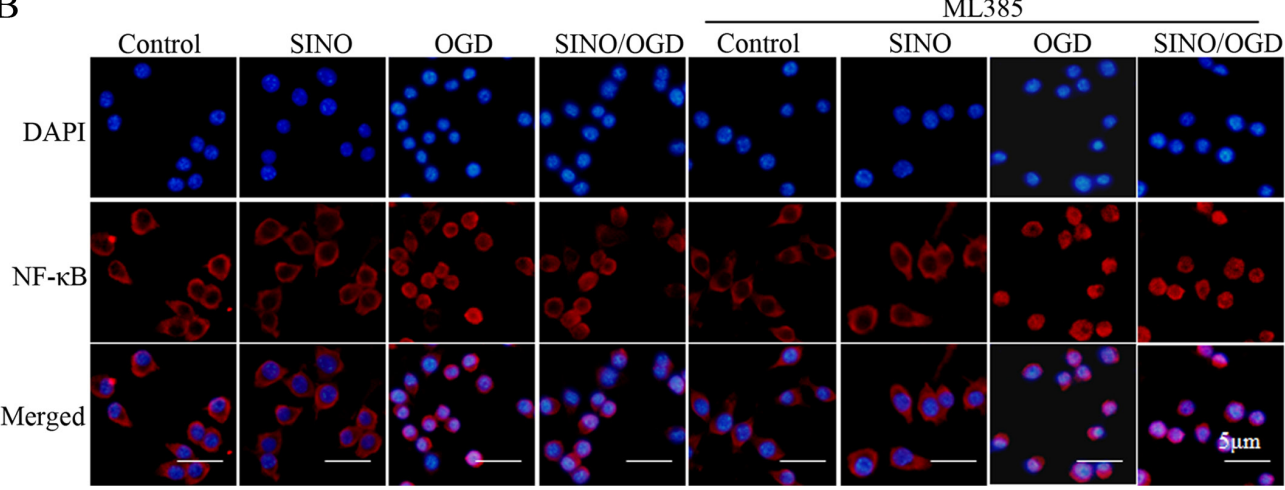

Figure 5. SINO treatment regulates microglia inflammation in an Nrf2-dependent manner. (A) BV2 cells were pretreated with ML385 (5 $\mu \mathrm{M}$ ) for $48 \mathrm{~h}$ to inhibit Nrf 2 expression. Cells were then stimulated with OGD for $4 \mathrm{~h}$ followed by treatment with SINO (200 $\mu \mathrm{M})$ for $12 \mathrm{~h}$. The protein expression levels of p-I $\mathrm{B} \alpha$ and total $\mathrm{I} \kappa \mathrm{B} \alpha$ were analyzed using western blotting. (B) Immuno-fluorescent staining of cells was performed using an NF- $\mathrm{B}$ p65 primary antibody and Dylight 488 conjugated secondary antibody (middle row of panels). The cells were also stained with DAPI (top row of panels) and merged with NF- $\kappa \mathrm{B}$ images (lower row of panels). The data are presented as the mean \pm SEM of three independent experiments. ${ }^{* *} \mathrm{P}<0.01$ vs. Control; ${ }^{\# \#} \mathrm{P}<0.01 \mathrm{vs}$. OGD. OGD, oxygen and glucose deprivation; p-, phosphorylated; SINO, sinomenine.

upregulation of the expression levels of M1 markers, IL-6 and NOS2, while it markedly upregulated the expression levels of the M2 markers, Arg-1 and IL-10, suggesting that SINO may have dual functions in modulating microglia polarization.

Accumulating evidence has revealed that Nrf2 serves a crucial role behind the processes of oxidative stress and inflammation $(50,51)$. Therefore, Nrf2 may represent a promising target for stroke intervention (50). However, the present understanding of the functions of Nrf 2 and its application for stroke-targeted therapy remains limited (50). In the present study, SINO was demonstrated to protect against cerebral injury in MCAO mice. The protective effects were associated with the ability of SINO to activate the Nrf2 signaling pathway. The functional importance of Nrf2 has been reported in several pathological conditions, most of which are from studies performed investigating Nrf2-/- mice $(52,53)$. Protopanaxtriols, as a potent Nrf2 natural inducer extracted from the root of Panax ginseng, C.A. Mey. has been widely used in East Asia for thousands of years, exhibiting potent anti-inflammatory and antioxidative properties $(54,55)$. Previous research has reported that Nrf2 deficiency causes a significant increase in infarct volume following MCAO for 3 days, which indicates that $\mathrm{Nrf} 2$ may exert a beneficial role in ischemic injury during the acute development and progression (56-58). The findings of the present study confirmed that SINO treatment effectively reversed the MCAO-associated induction of the inflammatory cytokines, TNF- $\alpha$ and IL- $1 \beta$, and the activities of the antioxidant enzymes, GPx and SOD. In addition, the SINO-induced activation of Nrf2 signaling was found to be essential for SINO-induced cerebral protection, as the inhibition of Nrf2 eliminated the protective effects of SINO on MCAO-associated cerebral injury. In theory, the activation of Nrf2 should upregulate SOD; however, in the current study, the enzymatic activity of SOD in the brains of MCAO model mice was found to be decreased and Nrf2 was induced to act against brain tissue damage in MCAO model mice. Therefore, the light upregulation of Nrf2 in MCAO model mice may not prevent the downregulation of SOD.

According to a previous study, the Nrf2-target genes were revealed to be preferentially activated in glial cells, which produces a more effective antioxidant effect than neurons (59). Over the past decade, previous findings have suggested that SINO may exert a significant potential for the treatment of strokes (27,60-62). Glial cells contribute to the expansion and resolution of infarctions, and affect the process of ischemic injury. However, the long-term effects of Nrf2 function and SINO neuroprotection on ischemic injury remain unclear (50). Previous studies have revealed the mechanism of Nrf2 pathway $(63,64)$. The present study suggested that SINO-induced regulation of Nrf2 signaling may be an important pathway through which SINO protects against MCAO-associated cerebral injury, although the precise mechanism of action requires further investigation.

In conclusion, the findings of the present study suggested that the SINO-induced regulation of Nrf2 signaling may be an important pathway through which SINO protects against MCAO-associated cerebral injury. The results indicated that SINO treatment may activate Nrf2 in microglia and subsequently modulate microglia polarization. The SINO-induced inhibition of M1 polarization and promotion of M2 polarization in microglia contributed to its anti-inflammatory and cerebral protective properties. Since the current MCAO model shares its pathogenesis with numerous other cerebral diseases, these findings may provide novel insights into the potential application of SINO for the treatment of brain or other inflammatory diseases. 


\section{Acknowledgements}

Not applicable.

\section{Funding}

The present study was supported by a grant from the Natural Science Basic Research Program of Shaanxi (grant no. 2018JM7140).

\section{Availability of data and materials}

The datasets used and/or analyzed during the current study are available from the corresponding author on reasonable request.

\section{Authors' contributions}

FB, YZ and WL carried out the experimental work, as well as the data collection and interpretation. FB and WL participated in the design and coordination of experimental work and acquisition of data. WL and KX carried out the study design; the analysis and interpretation of data; and drafted the manuscript. WL and KX confirmed the authenticity of all the raw data. All authors read and approved the final manuscript.

\section{Ethics approval and consent to participate}

The present study was approved by the Ethics Committee of the Medical College of Xi'an Peihua University.

\section{Patient consent for publication}

Not applicable.

\section{Competing interests}

The authors declare that they have no competing interests.

\section{References}

1. Jeon JH, Jung HW, Jang HM, Moon JH, Park KT, Lee HC, Lim HY, Sur JH, Kang BT, Ha J and Jung DI: Canine model of ischemic stroke with permanent middle cerebral artery occlusion: Clinical features, magnetic resonance imaging, histopathology, and immunohistochemistry. J Vet Sci 16: 75-85, 2015.

2. Ito M, Shichita T, Okada M, Komine R, Noguchi Y, Yoshimura A and Morita R: Bruton's tyrosine kinase is essential for NLRP3 inflammasome activation and contributes to ischaemic brain injury. Nat Commun 6: 7360, 2015.

3. Fu Y, Liu Q, Anrather J and Shi FD: Immune interventions in stroke. Nat Rev Neurol 11: 524-535, 2015.

4. Pont-Lezica L, Bechade C, Belarif-Cantaut Y, Pascual O and Bessis A: Physiological roles of microglia during development. J Neurochem 119: 901-908, 2011.

5. Subhramanyam CS, Wang C, Hu Q and Dheen ST: Microgliamediated neuroinflammation in neurodegenerative diseases. Semin Cell Dev Biol 94: 112-120, 2019.

6. Xu L, He D and Bai Y: Microglia-Mediated inflammation and neurodegenerative disease. Mol Neurobiol 53: 6709-6715, 2016.

7. Dong YF, Chen ZZ, Zhao Z, Yang DD, Yan H, Ji J and Sun XL: Potential role of microRNA-7 in the anti-neuroinflammation effects of nicorandil in astrocytes induced by oxygen-glucose deprivation. J Neuroinflammation 13: 60, 2016.

8. Qin C, Zhou LQ, Ma XT, Hu ZW, Yang S, Chen M, Bosco DB, Wu LJ and Tian DS: Dual functions of microglia in ischemic stroke. Neurosci Bull 35: 921-933, 2019.
9. Skowron MA, Niegisch G, Albrecht P, van Koeveringe G, Romano A, Albers P, Schulz WA and Hoffmann MJ: Various mechanisms involve the nuclear factor (Erythroid-Derived 2)Like (NRF2) to achieve cytoprotection in long-term cisplatin-treated urothelial carcinoma cell lines. Int J Mol Sci 18: $1680,2017$.

10. Fao L, Mota SI and Rego AC: Shaping the Nrf2-ARE-related pathways in Alzheimer's and Parkinson's diseases. Ageing Res Rev 54: 100942, 2019.

11. Robledinos-Anton N, Fernandez-Gines R, Manda G and Cuadrado A: Activators and Inhibitors of NRF2: A review of their potential for clinical development. Oxid Med Cell Longev 2019: 9372182, 2019.

12. Song S, Shen X, Tang Y, Wang Z, Guo W, Ding G, Wang Q and $\mathrm{Fu} \mathrm{Z}$ : Sinomenine pretreatment attenuates cold ischemia/reperfusion injury in rats: The role of heme oxygenase-1. Int Immunopharmacol 10: 679-684, 2010.

13. Ko WC, Shieh JM and Wu WB: P38 MAPK and Nrf2 activation mediated naked gold nanoparticle induced heme oxygenase-1 expression in rat aortic vascular smooth muscle cells. Arch Med Res 51: 388-396, 2020.

14. Zhao Z, Xiao J, Wang J, Dong W, Peng Z and An D: Anti-inflammatory effects of novel sinomenine derivatives. Int Immunopharmacol 29: 354-360, 2015.

15. Cheng Y,Li F, Wang D, Zhang Y, Yuan F and Zhang J: Sinomenine inhibits the expression of PD-L1 in the peripheral blood mononuclear cells of mesangial proliferative nephritis patients. Mol Med Rep 7: 1223-1228, 2013.

16. Shen Q, Zhang X, Qi J, Shu G, Du Y and Ying X: Sinomenine hydrochloride loaded thermosensitive liposomes combined with microwave hyperthermia for the treatment of rheumatoid arthritis. Int J Pharm 576: 119001, 2020.

17. Tang J, Raza A, Chen J and Xu H: A systematic review on the sinomenine derivatives. Mini Rev Med Chem 18: 906-917, 2018.

18. Wang Q and Li XK: Immunosuppressive and anti-inflammatory activities of sinomenine. Int Immunopharmacol 11: 373-376, 2011.

19. Jiang Y, Gao M, Wang W, Lang Y, Tong Z, Wang K, Zhang H, Chen G, Liu M, Yao Y and Xiao X: Sinomenine hydrochloride protects against polymicrobial sepsis via autophagy. Int J Mol Sci 16: 2559-2573, 2015

20. Qian L, Xu Z, Zhang W, Wilson B, Hong JS and Flood PM: Sinomenine, a natural dextrorotatory morphinan analog, is anti-inflammatory and neuroprotective through inhibition of microglial NADPH oxidase. J Neuroinflammation 4: 23, 2007.

21. Sun Y, Yao Y and Ding CZ: A combination of sinomenine and methotrexate reduces joint damage of collagen induced arthritis in rats by modulating osteoclast-related cytokines. Int Immunopharmacol 18: 135-141, 2014.

22. Liu S, Chen Q, Liu J, Yang X, Zhang Y and Huang F: Sinomenine protects against $E$. coli-induced acute lung injury in mice through Nrf2-NF-кB pathway. Biomed Pharmacother 107: 696-702, 2018.

23. Wang Y, Yu C and Zhang H: Lipopolysaccharides-mediated injury to chondrogenic ATDC5 cells can be relieved by Sinomenine via downregulating microRNA-192. Phytother Res 33: 1827-1836, 2019.

24. Bickler PE, Clark JP, Gabatto P and Brosnan H: Hypoxic preconditioning and cell death from oxygen/glucose deprivation co-opt a subset of the unfolded protein response in hippocampal neurons. Neuroscience 310: 306-321, 2015.

25. Liu Y, Lu L, Hettinger CL, Dong G, Zhang D, Rezvani K, Wang X and Wang H: Ubiquilin-1 protects cells from oxidative stress and ischemic stroke caused tissue injury in mice. J Neurosci 34: 2813-2821, 2014

26. Wu WN, Wu PF, Chen XL, Zhang Z, Gu J, Yang YJ, Xiong QJ, $\mathrm{Ni}$ L, Wang F and Chen JG: Sinomenine protects against ischaemic brain injury: Involvement of co-inhibition of acid-sensing ion channel 1a and L-type calcium channels. Br J Pharmacol 164: 1445-1459, 2011.

27. Qiu J, Yan Z, Tao K, Li Y, Li Y, Li J, Dong Y, Feng D and Chen H: Sinomenine activates astrocytic dopamine D2 receptors and alleviates neuroinflammatory injury via the CRYAB/STAT3 pathway after ischemic stroke in mice. J Neuroinflammation 13: 263, 2016.

28. Bi F, Chen F, Li Y, Wei A and Cao W: Klotho preservation by Rhein promotes toll-like receptor 4 proteolysis and attenuates lipopolysaccharide-induced acute kidney injury. J Mol Med (Berl) 96: 915-927, 2018.

29. Wang B, Tian S, Wang J, Han F, Zhao L, Wang R, Ning W, Chen $\mathrm{W}$ and $\mathrm{Qu} \mathrm{Y}$ : Intraperitoneal administration of thioredoxin decreases brain damage from ischemic stroke. Brain Res 1615: 89-97, 2015. 
30. Qin T, Du R, Huang F, Yin S, Yang J, Qin S and Cao W: Sinomenine activation of Nrf2 signaling prevents hyperactive inflammation and kidney injury in a mouse model of obstructive nephropathy. Free Radic Biol Med 92: 90-99, 2016.

31. Livak KJ and Schmittgen TD: Analysis of relative gene expression data using real-time quantitative PCR and the 2(-Delta Delta C(T)) method. Methods 25: 402-408, 2001.

32. Yin S and Cao W: Toll-like receptor signaling induces Nrf2 pathway activation through p62-Triggered Keap1 degradation. Mol Cell Biol 35: 2673-2683, 2015.

33. Liu Q, Zhang F, Zhang X, Cheng R, Ma JX, Yi J and Li J: Fenofibrate ameliorates diabetic retinopathy by modulating Nrf2 signaling and NLRP3 inflammasome activation. Mol Cell Biochem 445: 105-115, 2018

34. Wan JJ, Wang PY, Zhang Y, Qin Z, Sun Y, Hu BH, Su DF, Xu DP and Liu X: Role of acute-phase protein ORM in a mice model of ischemic stroke. J Cell Physiol 234: 20533-20545, 2019.

35. Zhang F, Zhang JG, Yang W, Xu P, Xiao YL and Zhang HT: 6-Gingerol attenuates LPS-induced neuroinflammation and cognitive impairment partially via suppressing astrocyte overactivation. Biomed Pharmacother 107: 1523-1529, 2018.

36. Zalewska-Ziob M, Adamek B, Kasperczyk J, Romuk E, Hudziec E, Chwalińska E, Dobija-Kubica K, Rogoziński P and Bruliński K: Activity of antioxidant enzymes in the tumor and adjacent noncancerous tissues of non-small-cell lung cancer. Oxid Med Cell Longev 2019: 2901840, 2019.

37. Virani SS, Alonso A, Benjamin EJ, Bittencourt MS Callaway CW, Carson AP, Chamberlain AM, Chang AR, Cheng S, Delling FN, et al: Heart disease and stroke statistics-2020 update: A report from the American heart association. Circulation 141: e139-e596, 2020.

38. Kernan WN, Ovbiagele B, Black HR, Bravata DM, Chimowitz MI Ezekowitz MD, Fang MC, Fisher M, Furie KL, Heck DV, et al: Guidelines for the prevention of stroke in patients with stroke and transient ischemic attack: A guideline for healthcare professionals from the American heart association/American stroke association. Stroke 45: 2160-2236, 2014.

39. Go AS, Mozaffarian D, Roger VL, Benjamin EJ, Berry JD, Blaha MJ, Dai S, Ford ES, Fox CS, Franco S, et al: Heart disease and stroke statistics-2014 update: A report from the American heart association. Circulation 129: e28-e292, 2014.

40. Khoshnam SE, Winlow W, Farbood Y, Moghaddam HF and Farzaneh M: Emerging roles of microRNAs in ischemic stroke: As possible therapeutic agents. J Stroke 19: 166-187, 2017.

41. Li WX, Qi F, Liu JQ, Li GH, Dai SX, Zhang T, Cheng F, Liu D and Zheng SG: Different impairment of immune and inflammation functions in short and long-term after ischemic stroke. Am J Transl Res 9: 736-745, 2017.

42. Cowan M and Petri WA Jr: Microglia: Immune regulators of neurodevelopment. Front Immunol 9: 2576, 2018.

43. Lee JH, Wei ZZ, Cao W, Won S, Gu X, Winter M, Dix TA, Wei L and Yu SP: Regulation of therapeutic hypothermia on inflammatory cytokines, microglia polarization, migration and functional recovery after ischemic stroke in mice. Neurobiol Dis 96: 248-260, 2016.

44. Wu HY, Tang XQ, Liu H, Mao XF and Wang YX: Both classic Gs-cAMP/PKA/CREB and alternative Gs-cAMP/PKA/ p38beta/CREB signal pathways mediate exenatide-stimulated expression of M2 microglial markers. J Neuroimmunol 316: $17-22,2018$

45. Wu HY, Tang XQ, Mao XF and Wang YX: Autocrine interleukin-10 mediates glucagon-like peptide-1 receptor-induced spinal microglial $\beta$-endorphin expression. J Neurosci 37 : 11701-11714, 2017.

46. Miao H, Li R, Han C, Lu X and Zhang H: Minocycline promotes posthemorrhagic neurogenesis via M2 microglia polarization via upregulation of the TrkB/BDNF pathway in rats. J Neurophysiol 120: 1307-1317, 2018.
47. Dai J, Ding Z, Zhang J, Xu W, Guo Q, Zou W, Xiong Y, Weng Y, Yang Y, Chen S, et al: Minocycline relieves depressive-like behaviors in rats with bone cancer pain by inhibiting microglia activation in hippocampus. Anesth Analg 129: 1733-1741, 2019.

48. Michels M, Abatti MR, Avila P, Vieira A, Borges H, Carvalho C Jr, Wendhausen D, Gasparotto J, Tiefensee Ribeiro C, Moreira JC, et al: Characterization and modulation of microglial phenotypes in an animal model of severe sepsis. J Cell Mol Med 24: 88-97, 2020.

49. Kata D, Foldesi I, Feher LZ, Hackler L Jr, Puskas LG and Gulya K: Rosuvastatin enhances anti-inflammatory and inhibits pro-inflammatory functions in cultured microglial cells. Neuroscience 314: 47-63, 2016.

50. Liu L, Locascio LM and Dore S: Critical role of Nrf2 in experimental ischemic stroke. Front Pharmacol 10: 153, 2019.

51. Zhang R, Xu M, Wang Y, Xie F, Zhang G and Qin X: Nrf2-a promising therapeutic target for defensing against oxidative stress in stroke. Mol Neurobiol 54: 6006-6017, 2017.

52. Hayes JD and Dinkova-Kostova AT: The Nrf2 regulatory network provides an interface between redox and intermediary metabolism. Trends Biochem Sci 39: 199-218, 2014.

53. Benarroch EE: Nrf2, cellular redox regulation, and neurologic implications. Neurology 88: 1942-1950, 2017.

54. Rajabian A, Rameshrad M and Hosseinzadeh H: Therapeutic potential of Panax ginseng and its constituents, ginsenosides and gintonin, in neurological and neurodegenerative disorders: A patent review. Expert Opin Ther Pat 29: 55-72, 2019.

55. Kim KH, Lee D, Lee HL, Kim CE, Jung K and Kang KS: Beneficial effects of Panax ginseng for the treatment and prevention of neurodegenerative diseases: Past findings and future directions. J Ginseng Res 42: 239-247, 2018.

56. Liu L, Vollmer MK, Fernandez VM, Dweik Y, Kim H and Dore S: Korean red ginseng pretreatment protects against long-term sensorimotor deficits after ischemic stroke likely through Nrf2. Front Cell Neurosci 12: 74, 2018.

57. Liu L, Vollmer MK, Ahmad AS, Fernandez VM, Kim H and Dore S: Pretreatment with Korean red ginseng or dimethyl fumarate attenuates reactive gliosis and confers sustained neuroprotection against cerebral hypoxic-ischemic damage by an Nrf2-dependent mechanism. Free Radic Biol Med 131: 98-114, 2019.

58. Shih AY, Li P and Murphy TH: A small-molecule-inducible Nrf2-mediated antioxidant response provides effective prophylaxis against cerebral ischemia in vivo. J Neurosci 25: 10321-10335, 2005

59. Vargas MR and Johnson JA: The Nrf2-ARE cytoprotective pathway in astrocytes. Expert Rev Mol Med 11: e17, 2009.

60. Yang S, Ning F, Li J, Guo D, Zhang L, Cui R and Liu Y: Therapeutic effect analysis of sinomenine on rat cerebral ischemia-reperfusion injury. J Stroke Cerebrovasc Dis 25: 1263-1269, 2016

61. Qiu J, Wang M, Zhang J, Cai Q, Lu D, Li Y, Dong Y, Zhao T and Chen $\mathrm{H}$ : The neuroprotection of Sinomenine against ischemic stroke in mice by suppressing NLRP3 inflammasome via AMPK signaling. Int Immunopharmacol 40: 492-500, 2016.

62. Rastogi V, Santiago-Moreno J and Dore S: Ginseng: A promising neuroprotective strategy in stroke. Front Cell Neurosci 8: 457, 2015.

63. Bartolini D, Dallaglio K, Torquato P, Piroddi M and Galli F: Nrf2-p62 autophagy pathway and its response to oxidative stress in hepatocellular carcinoma. Transl Res 193: 54-71, 2018.

64. Sun X, Chen L and He Z: PI3K/Akt-Nrf2 and anti-inflammation effect of macrolides in chronic obstructive pulmonary disease. Curr Drug Metab 20: 301-304, 2019.

This work is licensed under a Creative Commons Attribution-NonCommercial-NoDerivatives 4.0 International (CC BY-NC-ND 4.0) License. 